\title{
Investigating the influence of contextual factors in the coordination of chronic mental illness care in a district health system
}

\author{
Stanford Mandlenkosi Phehlukwayo ${ }^{1}$, Joyce Mahlako Tsoka-Gwegweni ${ }^{2}$
}

1. School of Health Sciences, College of Health Sciences,University of KwaZulu-Natal, Durban, South Africa. Private Bag X54001, Durban, 4000. Tel: +27 312607954 Mobile: +27 73451 9004; Email: phehlukwayos@ukzn.ac.za 2. School of Nursing and Public Health, College of Health Sciences, University of KwaZulu-Natal, Durban, South Africa. Correspondence: Email: tsokagwegweni@ukzn.ac.za

\begin{abstract}
Background: The global shift from institution-based to community-based care for chronic mental illness (CMI) care resulted in the de-institutionalization of clients with CMI. However, health systems which have been originally designed for acute hospital-based care do not seem to be appropriately transformed to manage CMI care at a community level.

Aim: The aim of this study was to investigate how contextual factors influenced care coordination for chronic mental illness care within the eThekwini District.

Methods: This study employed a qualitative multiple case study design with instrumental approach. Maximum variation sampling was used to select five Community Health Centres (CHC's) and 48 health providers who worked with mentally ill clients in the sample CHC's. Framework analysis was used to analyze the results.

Results: Inequitably resourced catchment areas, unclear referral systems, high staff turnover, freezing of posts, chronic staff shortage and adverse working conditions contributed to care fragmentation, poor client care and client loss in the system. On the other hand, limited community support systems constituted barriers for client reintegration into society and relapses.

Conclusion: The study concluded that the eThekwini District health facility settings were not adequately equipped to respond to care coordination demands for chronic mental illness care.

Keywords: De-institutionalization, health facility settings, community support services, chronic mental Illness.

DOI: https://dx.doi.org/10.4314/ahs.v18i4.24

Cite as: Phehlukwayo SM, Tsoka-Gwegweni JM. Investigating the influence of contextual factors in the coordination of chronic mental illness care in a district health system. Afri Health Sci. 2018;18(4): 1027-1035. https:/ / dx.doi.org/ 10.4314/ abs.v18i4.24
\end{abstract}

\section{Introduction}

The global policy shift from institution-based to community-based system of care for chronic illness culminated into the de-institutionalization of people with chronic illness, including individuals with chronic mental illness $(\mathrm{CMI})^{1}$. However, the health systems which have been

\section{Corresponding author:}

Stanford Mandlenkosi Phehlukwayo, School of Health Sciences, College of Health

Sciences, University of KwaZulu-Natal, Durban, South Africa. Private Bag X54001, Durban, 4000.

Tel: +27 312607954

Mobile: +27 73451 9004,

Email: phehlukwayos@ukzn.ac.za historically designed to respond to institution-based acute care seem to be poorly prepared to cope with the demand for community-based chronic illness care. When individuals in need of chronic mental illness care are de-institutionalized, healthcare tasks that were carried out by centralized hospital teams are shifted to a range of health service providers in community-based settings which are often fragmented, under-resourced and uncoordinated. According to the World Health Organization (WHO), the de-institutionalization of people with CMI is a complex process which must be accompanied by a network of alternative support structures outside the psychiatric institution ${ }^{1,2}$. Currently people with CMI, who have been discharged into their community often relapse and get readmitted into hospitals due to poorly planned de-institutionalization and lack of care coordination in community-based settings. 
Care coordination is a complex concept in that it is enmeshed in various aspects of health care. In essence, care coordination can be summarized as an integration of different services intended to manage a full range of care continuum across healthcare settings and between health service providers, patients and families ${ }^{3,4}$. The complexity of care coordination implies that poorly managed patient transitions between healthcare settings and between healthcare providers can result in care fragmentation and an increase in the risk of non-compliance to treatment and re-admissions to hospital. Although empirical evidence shows that care coordination is associated with better managed client transition between healthcare settings and health care providers, there is still limited attention paid to care coordination. Research shows that care coordination leads to compliance to medication, better symptom control, improved mental health, improved functioning and decreased emergency re-hospitalizations ${ }^{5}$.

Worldwide, mental disorders continue to contribute significantly to the burden of disease and disability with alarming statistics which have been projected for the next decade. In common psychotic conditions like schizophrenia, relapses are characterized by acute psychotic episodes which are accompanied by personal risk of self-harm; social risk of danger to others, biological risk of disease progression and deterioration in the level of function ${ }^{6}$. The deterioration in clients' level of function reduces their capacity to manage the condition which is accompanied by the burden of care.

Statistical records indicate that mental disorders account for nearly $12 \%$ of the global burden of disease and it is projected that mental illness will account for about 15\% of the disability-adjusted life years (DALY) lost to illness by $2020^{7,8}$. In addition, mental disorders which include schizophrenia, mood disorders, substance abuse and dementia are often co-morbid with many physical health problems such as cancer, HIV/AIDS, diabetes and tuberculosis 9 . In cases where mental conditions are co-morbid with physical conditions, mental conditions are often marginalized because they are less overt or perceived as not life threatening. This marginalization of mental illness further exacerbates the burden of illness.
Locally, the KwaZulu-Natal Province (KZNP) still faces the task of uniting the structurally, functionally and geographically fragmented health care system inherited from the previous health care system ${ }^{10}$. In the KZNP, psychosis alone ranks number 15 in the leading diagnoses seen in the public health facilities ${ }^{11}$. A combination of the marginalization of mental conditions which are comorbid with physical conditions and the historical backlog of fragmented care continues to contribute to the insidious increase in the burden of mental illness and the burden of disease.

The above background indicates that health systems do not seem to be adequately prepared to cope with de-institutionalized clients in community settings. The de-institutionalization process seems to be poorly planned and community based settings do not seem to be adequately resourced to cope with chronic illness care. Chronic mental illness care seems to be fragmented and poorly coordinated with resultant increase in the relapse rate, hospital re-admissions, burden of care and the burden of disease. This is indicative of a health system gap for chronic mental illness care at a DHS level. The purpose of this study was to investigate how contextual factors influenced care coordination for chronic mental illness within a DHS.

This paper constitutes Paper 2 of the main dissertation which investigated the healthcare system elements for CMI care coordination in the e'Thekwini District. The health care settings and the community support services constituted the care coordination context in this study ${ }^{12}$.

\section{Methods \\ The Study area}

This study was carried out in the e-Thekwini District which is located in the KZNP of the Republic of South Africa. The high population density, the historically fragmented healthcare services and the geographically diverse catchment areas served by the District provided the unique contextual elements for investigating CMI care coordination in this District.

\section{Study design}

A descriptive instrumental multiple case study design 
was utilized for this study ${ }^{13}$. Purposive maximum variation sampling strategy was used to select the Community Health Centres (CHC's) and the key informants for this study ${ }^{14,15}$. Maximum variation allowed the selection of a combination of key informants from diverse geographi- cal settings with diverse professional background that was likely to impact on participants' views on contextual factors which influenced care coordination ${ }^{16}$. A sample of five CHC's which serviced geographically diverse catchment areas in the North, South and West sub-Districts was selected as illustrated in Table 1.

Table 1: CHC Profile

\begin{tabular}{|l|c|l|l|}
\hline SUB-DISTRICT & \multicolumn{1}{|l|}{$\begin{array}{l}\text { SAMPLE } \\
\text { SIZE }\end{array}$} & CONTROL AUTHORITY & GEO. LOCATION \\
\hline South Sub-District & 1 & Provincial \& Local Authority & Semi-Urban \& Informal Settlement \\
\hline \multirow{3}{*}{ North Sub-District } & \multirow{2}{*}{3} & Provincial Authority & Urban \& Semi-Urban \\
\cline { 3 - 4 } & & Provincial Authority & Semi-Urban \& Rural \\
\cline { 3 - 4 } & & Provincial Authority & Semi-Urban \& Informal Settlement \\
\hline West Sub-District & 1 & Provincial Authority & Semi-Urban \& Rural \\
\hline
\end{tabular}

A sample of 48 key informants was drawn from various health disciplines who worked with CMI clients in the five CHC's. The sample size for key informants was based on the minimum sample size guidelines for reaching saturation in qualitative research which ranged from 12-30 participants $^{14,18}$. See Table 2.

Table 2: The Key Informant Sample Profile $(\mathrm{N}=48)$

\begin{tabular}{|c|c|c|c|c|c|}
\hline $\begin{array}{c}\text { SERVICE PROVIDER } \\
(\mathrm{n}=\% \mathrm{~N})\end{array}$ & $\begin{array}{c}\text { CHC A } \\
\text { Semi-Urban \& } \\
\text { Inf. Settlement } \\
(\mathrm{n}=10)\end{array}$ & $\begin{array}{c}\text { CHC B } \\
\text { Urban \& } \\
\text { Semi-Urban } \\
(\mathrm{n}=11)\end{array}$ & $\begin{array}{c}\text { CHC C } \\
\text { Urban \& } \\
\text { Semi-Urban } \\
(\mathrm{n}=10)\end{array}$ & $\begin{array}{c}\text { CHC D } \\
\text { Semi-Urban } \\
\text { \& Informal } \\
\text { Settlement } \\
(\mathrm{n}=10)\end{array}$ & $\begin{array}{c}\text { CHC E } \\
\text { Semi-Urban } \\
\text { \& Rural } \\
(\mathrm{n}=7)\end{array}$ \\
\hline Clinical psychologist & 1 & 1 & 1 & 0 & 0 \\
\hline Community caregiver & 7 & 6 & 6 & 6 & 5 \\
\hline Medical officer & 0 & 0 & 1 & 0 & 0 \\
\hline Nurse & 1 & 2 & 2 & 2 & 1 \\
\hline Occupational therapist & 1 & 1 & 0 & 1 & 1 \\
\hline Social worker & 0 & 1 & 0 & 1 & 0 \\
\hline
\end{tabular}

\section{Ethical approval and ethical consideration}

Ethical approval for this study was obtained from the University of KwaZulu-Natal Biomedical Research and Ethics Committee (REF: BE 323/14). Gatekeeper permission to conduct this study was obtained from the eThekwini District Manager, the KwaZulu-Natal Province Health Research Committee (REF. HRKM 294/14) and the Health Facility Managers from all the CHCs who formed the study sample. All prospective participants were invited to participate in the study and provided with information on what the study entailed, which included the study benefits, their rights to participate or refuse to participate or withdraw anytime from the study as well as issues regarding privacy, confidentiality and anonymity. Prior to the commencement of data collection, all participants were required to sign an Informed Consent Form 
confirming their willingness to participate in the study. During data collection all interviews were conducted in an isolated room to ensure privacy. The study sample CHCs were allocated alphabetic codes and Key Informants were allocated numerical codes to ensure anonymity. During the interviews, all identifying information directly linking $\mathrm{CHCs}$ or key informants to the content of interviews was omitted or deleted from the transcripts, audio recording, the main report and all publications or presentations of this study to ensure anonymity. During data analysis, all hard copies of transcripts, field notes, CHC Health documents accessed during data collection and electronic data were kept in a locked cupboard and stored in a password protected computer respectively to ensureprivacyandconfidentiality.

All hard copies electronic raw data used for this study will be deleted or shredded after a period of five years of completion of thisstudy.

\section{Trustworthiness}

In order to ensure research rigor in this study, the research adopted the four criteria for addressing trustworthiness as proposed by Guba in Shenton (2004) namely, transferability, dependability, confirmability and credibility $^{19-21}$. In this study design, transferability was ensured by a detaileddescription of the study setting (See Table 1 above) and a clear description of the study sample (See Table 2) using 'thick description'. During data collection, dependability was achieved by a detailed documentation of the processes employed in the study and by developing the case study data base which could be reviewed by others to enable future researchers to emulate the same study. In order to ensure confirmability, the researcher took necessary steps to demonstrate that the findings had emerged from collected data and not from own predispositions. During data analysis credibility was achieved by looking for patterns in the data and across the data through pattern matching, and by comparing and contrasting dataacross respondents and data sources through triangulation. The recognition of the study limitations and their potential influence on the results, the in-depth description of the research methodology to allow for scrutiny of the research results integrity and the use of a diagram to illustrate the audit trail of the research process was used to reduce the researchers' bias.

\section{Data collection}

Data collection was conducted by the Principal Investigator (PI) using one-on-one semi-structured interviews. Each interview lasted up to 45 minutes and data was collected over a period of one month from 08 December 2014 to 15 January 2015. Prior to data collection, the Interview Question Schedule was piloted on the key informants in a similar CHC which was not included in the study sample. All interviews were audio-taped and backed up with a summary of participant responses, field notes and direct observations of physical settings which were compiled immediately after each interview as outlined in Table 3.

\section{Data analysis}

Framework analysis (FA) using deductive reasoning was used to analyze the results. FA involves five stages of data handling namely, familiarization; development of an analytic framework; indexing and charting; summarizing data in an analytic framework and synthetizing data by mapping and interpreting ${ }^{22,23,24}$. A total of three sub-themes emerged from the health facility settings theme namely, poor referral system and client cross-border migrations; poor health facility design and poor working conditions; and, high workloads. Poor Social Welfare support services; crime and emergency service disruption; socio-cultural and socio-economic factors constituted the community support services sub- themes. 
Table 3: Data Collection Plan

\begin{tabular}{|c|c|}
\hline $\begin{array}{l}\text { CARE } \\
\text { COORDINATION } \\
\text { DOMAIN }\end{array}$ & Care Coordination Contextual Factors \\
\hline STUDY OBJECTIVE & $\begin{array}{l}\text { To investigate the influence of contextual factors on the care coordination of chronic } \\
\text { mental illness care in a District Health System }\end{array}$ \\
\hline $\begin{array}{l}\text { DATA } \\
\text { COLLECTION } \\
\text { METHOD }\end{array}$ & $\begin{array}{l}\text { - Semi-structured interviews } \\
\text { - Audiorecording } \\
\text { - Observations \& Field notes }\end{array}$ \\
\hline $\begin{array}{l}\text { DATA } \\
\text { COLLECTION } \\
\text { SOURCE }\end{array}$ & Mental Health Service Providers \\
\hline $\begin{array}{l}\text { SEMI- } \\
\text { STRUCTURED } \\
\text { INTERVIEW } \\
\text { SCHEDULE }\end{array}$ & $\begin{array}{l}\text { Health Care Settings \& Community Support System } \\
\text { 1. Health Care Settings } \\
\text { - What is your opinion on your community based health settings for chronic illness } \\
\text { (CMI) care in theDistrict? } \\
\text { - Please describe the procedure followed when clients are discharged from } \\
\text { Hospital into community. } \\
\text { - What do you understand by care coordination? } \\
\text { 2. Community Support System } \\
\text { - What support services are available for CMI clients in yourcommunity? } \\
\text { - Do you get adequate support from management to adequately care for clients with } \\
\text { - WMI after discharge? } \\
\text { CMI after discharge? } \\
\text { Concluding Questions } \\
\text { - What would you change about current facility settings and or care for clients with } \\
\text { CMI in the District? }\end{array}$ \\
\hline
\end{tabular}

\section{Results}

This section presents results on the contextual factors which influenced the care coordination for chronic illness care based on the objectives of this study. The results are presented according to matching care coordination context elements from the study conceptual framework namely, the health facility settings and community support systems.

\section{Health facility settings}

Three sub-themes emerged from this theme namely, poor referral system and client cross-border migrations; poor health facility design and poor working conditions; and highworkload.

Poor referral system and client cross-border migrations

Overall, results showed that the District catchment ar- eas were inequitably resourced, fragmented and poorly planned. This was characterized by health service user (HSU) cross-border migrations between catchment areas and the neighbouring Districts, consultation of more accessible traditional medicine (TM) practitioners or confusion about the referral system amongst the HSUs.

"Our clientele is beyond our catchment areas. We serve clients from different catchment areas including other adjacent Health Districts" [Participant 5]

Poor health facility design and poor working conditions Observations showed that the health facility space was not appropriately designed to cater for service utilization and services provision. Poor signage or lack of signage made it difficult for clients to find their way around the facilities in some CHC's. Some staff members worked 
under poor and unsafe working conditions which compromised the quality of healthcare. In one $\mathrm{CHC}$, a health professional was allocated a medical examination cubicle as an office which doubled up as the "staff room" during lunch time. In the same $\mathrm{CHC}$, three senior staff members shared a standard size office for one person. [Field Notes].

In at least two other CHC's female staff held client consultations in confined office with psychiatric patients without any back-up security systems. [Field Notes].

At a community level, the CCG's indicated that at times they worked with clients who had communicable diseases which were difficult totreat.

"We work under risky conditions. Sometimes the defaulting client has MDR. It's easy for CCG's to contract MDR”. [Participant 20]

In another $\mathrm{CHC}$ the Psychiatric clinic was located in a separate isolated building which was also not accessible by public transport routes. This was repeatedly articulated as a barrier to the transfer and sharing of client information between healthcare service providers.

"We are one institution in different buildings and that affects our communication. You find that in this clinic we do not have access to client information from the psychiatric clinic [Main CHC]. You only rely on the referral letter from the referring Discipline". [Par- ticipant 5]

\section{High workload}

The high staff turnover combined with the freezing of posts frequently came up as a contributing factor for the chronic staff shortage and subsequent high workload. This negatively impacted on the continuity of care and poor quality of care for HSUs.

"Posts are frozen and do not get filled. This makes it difficult for people to stay in public health. This very discouraging and affects service delivery". [Participant 2]

"Patients are not motivated to attend the clinic...... Patients end up defaulting and getting lost in the system. .... when weeventually find the patient the patients will start complaining about poor ser-vice". [Participant19]

Community support services

Deficient basic Welfare Services, Crime and Emergency
Service Disruption, Socio-cultural factors and Stigma and Socio-economic factors constituted the sub-themes which emerged from this theme. The findings from this study showed that the community support services necessary for effective integration of clients with mental illness into society were inadequate and not conducive to effective care coordination.

\section{Deficient basic Social Welfare Services}

The CCGs often used their personal funds to provide food for clients who were waiting for delayed food parcels from Social Welfare or to assist clients with transport fare to go to the clinic to fetch medication.

"You also find you do not even have food powder to give clients before taking their medication. I sometimes leave money forpatients to buy food.......... Due to the delay of the food parcel process" [Participant 23].

\section{Crime and Emergency ambulance Services disruption}

The poor ambulance service was also exacerbated by security risks of Ambulance vehicle hijackings and other crime related risks especially in informal settlements.

"Ambulances notify us that their priority is emergencies for injured people....We do not get any refunds". [Participant 26] "Ambulance crew is concerned about their safety especially in an informal settlement. We end up using our money to provide transport'. [Participant 27]

\section{Socio-cultural factors and stigma}

The client's capacity to manage own condition was compromised by the nature of mental health condition combined with the clients' poor insight into the condition itself.

"In terms of carry over ... that becomes a problem especially for psych... most of them discontinue treatment because they can't cope with the change..." [Participant 3]

Some client admission procedures did not seem to take into account some accompanying risk factors which are associated with the mental condition itself.

"The suicidal and psychotic patients are emergency. You feel like you want to "do good" but the system won't allow you to do that". [Participant 2]

\section{Socio-cultural factors}

Some socio-cultural factors imbedded in some beliefs by certain families featured as the contributing factor in the disruption of client compliance to medication. 
"As well as [sic] in some households mental illness is regarded as taboo such that the person is hidden; which results in non-adherence to prescribed medication." [Participant 19]

The extended family bonds in the case of clients from informal settlements necessitated some occasional visits to relatives in remote rural areas for undefined duration of time which was often done without any arrangements with the CCG's or the clinic. This resulted in loss of clients in the system or non-compliance to treatment.

"After starting $\mathrm{Rx}$ with the clinic, some patients go back to their rural areas without notifying the clinic or requesting a referral letter for the local clinic". The patient ends up defaulting". [Participant 9]

\section{Discussion}

The main objective of this study was to investigate the contextual factors which influenced care coordination for chronic mental illness care within the eThekwini District. This discussion present the synthesis of the findings on the influence of health facility settings and community support services on the coordination of chronic illness care in a District HealthSystem.

\section{Health facility settings}

A combination of inequitably resourced catchment areas and confusion about the referral system invariably contributed to care fragmentation and loss of clients in the health system. These findings reflected the challenge still faced by the KwaZulu-Natal Province, of uniting a structurally, functionally and geographically fragmented health care system inherited from the previous healthcare system ${ }^{10}$. On the other hand, recent research shows that in low and middle income countries the consultation of TM practitioners remains high because it is embedded within contextual cultural and belief systems milieu, free from stigma and easily accessible ${ }^{26}$. In this study, the loss of clients in the system could be attributed to client consultation of TM practitioners who were more accessible and who understood the client culture.

Observations further indicated that some $\mathrm{CHC}$ layout were not appropriately designed to facilitate good quality of healthcare and staff safety. These findings were supported by recent research which showed that limited office space can impact negatively on client quality of care ${ }^{3,27}$. In spite of the research showing that working in isolation with mentally ill clients predisposed staff members to attacks by clients with mental illness, some staff members still worked in confined spaces without any backup security or support ${ }^{28}$. On the other hand, the occupational risk exposure to communicable diseases expressed by CCG's highlighted serious concerns about the working conditions of these healthcare service providers.

The continuous staff turnover and the freezing of posts directly contributed to the chronic staff shortage and high staff workload in the CHC's. This could be partly attributed to the Health District Human Resource Plan (HRP) which were not aligned to service delivery requirements and policy shift from institution based to community-based care ${ }^{11,29}$. According to Adnanes (2013), high staff turnover negatively influenced appropriate handover between health providers due to the nature and complexity of mental illness ${ }^{30}$. Similar studies identified stable services without breaks, seeing the same member of staff and coordination between different health providers as the pre-requisite for continuity of care ${ }^{30,31}$. A combination of these factors contributed to poor staff morale, the disruption of continuity of care or limited access to health care by clients.

\section{Community support systems}

This study further found that community support services (CSS) for chronic mental illness within the eThekwini District were still deficient. In terms of income support, entitlement and crisis response, the CCGs were faced with the burden of providing basic client subsistence and logistic support from their meager stipend due to delays in Disability Grant applications and the supply of food parcels. With regard to crisis support, poor ambulance service, failure to recognize mental illness as a priority and the security risk faced by the ambulance crew in informal settlements seriously compromised emergency response to client needs. These findings were supported by previous research which showed that the de-institutionalization of clients with CMI is often poorly planned and not accompanied by alternative support structures outside the psychiatric institution ${ }^{1,2,3}$

\section{Study Limitations}

Although the objectives of this study were achieved, researchers were aware of some unavoidable limitations which could be addressed in follow up studies and future research. Even though the findings from a case study can- 
not be generalized, the study design was rigorouslydocumented to facilitate possible replication of these findings in similar settings. The scope of this study was limited to service providers perspectives and related supportsystems due to the critical role of these components in care coordination for CMI. The findings from this study will provide a necessary context for future research on studies incorporating service user perspectives on care coordination for CMI within a DHS.

\section{Conclusion}

The study concluded that the historically inequitably resourced catchment areas and poorly communicated referral system contributed significantly to care fragmentation and loss of clients in the eThekwini District system. The poorly planned health facility space and the working environments in client households which posed occupational risks for staff impacted negatively on client quality of care. The continuous staff turnover, the freezing of posts and deficient community support systems contributed to chronic shortage of staff, care fragmentation and poor reintegration of clients into society. This was exacerbated by the socio-cultural factors, socio-economic factors and multiple health service provider consultations by clients which were not coordinated within client treatment milieu.

Overall, this study concluded that the eThekwini District care coordination context was not adequately equipped to respond sufficiently respond to care coordination demands for chronic mental illness care.

\section{Conflict of interest}

All authors declare no conflict of interest.

\section{References}

1. WHO. 'Organisation of Services for Mental Health' Mental Health Policy and Service Guidance Package. 2003.

2. Stein DJ, Allwood C, Em'sley RA. Community Care of Psychiatric Disorders in South Africa- Lessons from Research on Deinstitutionalisation. South African Medical Journal. 1999; 89.

3. McDonald KM, Schultz E, Albin L PN, Lonhart J, Sundaram V, Smith-Spangler C, et al. Care Coordination Atlas Version 4 American Institutes for Research;2014.

4. Miller K. Care Coordination Impacts on Access to

African Health Sciences Vol 18 Issue 4, December, 2018
Care for Children with Special Health Care Needs Enrolled in Medicaid and CHIP. Maternal \& Child Health Journal. 2014;18(4):864-72.

5. Joyce AS, Wild TC, Adair CE, McDougall GM, Gordon A, Costigan N, et al. Continuity of Care in Mental Health Services: Toward Clarifying the Construct. The Canadian Journal of Psychiatry / La Revue Canadienne de Psychiatrie. 2004;49(8):539-50.

6. Emsley R, Chiliza B, Asmal L, Harvey BH. The nature of relapse in schizophrenia. BMC Psychiatry. 2013;13:50.

7. Mental Health and work: Impact, issues and practices. [nternet]. Department of Mental Health and Substance Dependence Non-Communicable Diseases and Mental Health, WHO, Geneva.2002

8. WHO. WHO warns against mental illness. MedReview Report.;2004.

9. WHO. Integrating mental health services into primary health care Mental Health Policy, Planning and Service Development Information Sheet, Sheet 3. 2007

10. Harrison De. Health Care in KwaZulu-Natal : Implications for Planning. Health Systems Trust, Durban, SA; 1996.

11. Province DoHK-N. Strategic Plan 2010- 2014. Province DoHK-N; 2010 April2010.

12. McDonald K, Schultz E, Albin L, Pineda N, Lonhart J, Sundaram V, et al. Care coordination atlas version 3 (Prepared by Stanford University under subcontract to Battelle on Contract No. 290-04-0020). AHRQ Publication. 2010(110023EF).

13. Baxter P, Jack S. Qualitative case study methodology: Study design and implementation for novice researchers. The Qualitative Report. 2008;13(4):544-59.

14. Curry LA, Nembhard IM, Bradley EH. Qualitative and Mixed Methods Provide Unique Contributions to Outcomes Research. Circulation. 2009;119(10):1442 PubMed -52.

15. Nouria Brikci N, Green J. A guide to using qualitative research methodology. 2007.

16. Bricki N, Green J. A guide to using qualitative research methodology. 2007.

17. Nastasi B. Qualitative Research: Sampling \& Sample Size Considerations.u.d.

18. Onwuegbuzie AJ, Collins KM. A typology of mixed methods sampling designs in social science research. The Qualitative Report. 2007;12(2):281-316.

19. Shenton AK. Strategies for ensuring trustworthiness in qualitative research projects. Education for Information. 2004;22 (22):14. 
20. AgostinhoS. Naturalistic inquiry in e-learning research. International Journal of Qualitative Methods. 2004; 4(1), Article 2:13.

21. Gilson L. Health Policy and Systems Research: A Methodology Reader. Alliance for Health Policy and Systems Research, World Health Organization.2012.

22. Ward DJ, Furber C, Tierney S, Swallow V. Using framework analysis in nursing research: a worked example. Journal of Advanced Nursing. 2013; 69(11): 2423-31.

23. Srivastava A, Thomson SB. Framework analysis: a qualitative methodology for applied policy research. 2009. 24. Gale NK, Heath G, Cameron E, Rashid S,Redwood $\mathrm{S}$. Using the framework method for the analysis of qualitative data in multi-disciplinary health research. $B M C$ Medical Research Methodology. 2013;13(1):1-8.

25. Johnson C. The Group Areas Act-Stage I. S African LJ.1951;68:286.

26. Gureje O, Nortje G, Makanjuola V, Oladeji B, Seedat $\mathrm{S}$, Jenkins $\mathrm{R}$. The role of global traditional and comple- mentary systems of medicine in treating mental health problems. The Lancet Psychiatry. 2015; 2(2):168-77.

27. Ross CA, Goldner EM. Stigma, negative attitudes and discrimination towards mental illness within the nursing profession: a review of the literature. Journal of Psychiatric and Mental Health Nursing. 2009;16(6):558-67.

28. Cooper A, Mendonca J. A prospective study of patient assaults on nurses in a provincial psychiatric hospital in Canada. Acta Psychiatrica Scandinavica. 1991;84(2):163-6. 29. Health Do. White Paper for the Transformation of the Health sysytem in South Africa. In: Health Do, editor: Department of Health; 1997.

30. Adnanes M, Steihaug S. Obstacles to continuity of care in young mental health service users' pathways - an explorative study. International Journal Of Integrated Care. 2013;13:e031-e.

31. Metzger J. Preventing Hospital Readmissions: The First Test Case for Continuity of Care. Global Institute for Emerging Healthcare Practices. 2012:12. 